\title{
Mechanical Properties of Poly(lactic acid) Sheet Reinforced with Microfibrillated Cellulose from Corn Cobs
}

\author{
Prapatsorn Deejam ${ }^{1}$, Sireerat Charuchinda ${ }^{2, a}$ \\ ${ }^{1}$ Interdisciplinary Program in Petochemistry\& Polymer Science, Faculty of Science, Chulalongkorn University, \\ Bangkok 10330 Thailand \\ ${ }^{2}$ Department of Materials Science, Faculty of Science, Chulalongkorn University, Bangkok 10330 Thailand
}

\begin{abstract}
In this study, cellulose was extracted from corn cobs by successive hot $\mathrm{NaOH}$ solution and followed by $\mathrm{H}_{2} \mathrm{O}_{2}$ bleaching. XRD pattern show characteristic peak of Cellulose I. Microfibrillated cellulose (MFC) was successfully prepared by dissolving the extracted cellulose in $\mathrm{NaOH} /$ urea solution, shearing in a homogenizer and finally by freezing and thawing. To improve strength of MFC, MFC was physically crosslinked using PVA by freezing and thawing. The crosslinked MFC/PVA was added to poly(lactic acid) (PLA) to improve its mechanical properties. The non-crosslinked MFC/PVA was also prepared by only stirring the solution without freezing and thawing. MFC/PVA reinforced PLA films with various ratios of PLA and MFC/PVA at100:0, 99:1, 97:3 and 95:5were prepared through a solution casting method. Tensile strength and elongation at breakof PLA films increased with the addition of physically crosslinked MFC/PVA at $1 \% \mathrm{wt}$, whereas, the addition of non-crosslinked MFC/PVA decreased elongation at break. Crosslinking of MFC/PVA can improve tensile strength of PLA.It can render better tensile strength than that of non-crosslinked MFC/PVA. However, when MFC/PVA contents increase, tensile strength of PLA fims reinforced with non-crosslinked and crosslinked MFC/PVA decreased. Morphology of fracture surfaces reveals good dispersion and adhesion between 1\% crosslinked MFC/PVA and PLA matrix.
\end{abstract}

\section{Introduction}

Bio-based material has been attracted in both academic and industrial fields due to the growing concern about environmental protection and the rising cost of fossil fuel [1]. Nowadays, poly(lactic acid) (PLA) which is an aliphatic polyester made up of lactic acid (2-hydroxy propionic acid) [2] are widely used for bioplastic. The main limitations for application of PLA are their poor thermal and mechanical properties which can be improved by reinforcing with natural fibers such as microfibrillated cellulose (MFC) [3]. MFC is a cellulosic material, composed of expanded high-volume cellulose, greatly expanded in surface area and its diameter is in the range $20-60 \mathrm{~nm}$ with a length of several micrometers. It exhibits both amorphous and crystalline parts and presents a web-like structure that has a very good ability to form a rigid network [4]. Additionally, its properties can be improved with different methods such as grafting, chemical crosslinking and physical crosslinking [5].

Thus, the purpose of this study is to improve mechanical property of PLA reinforced with MFC. Since corn cobs are actually agricultural and feed industrial wastes in Thailand, in this study, cellulose was extracted from corn cobs by successive hot $\mathrm{NaOH}$ solution and followed by $\mathrm{H}_{2} \mathrm{O}_{2}$ bleaching. The MFC solution was prepared using $\mathrm{NaOH} /$ urea aqueous solution. To improve strength of MFC, MFC was then taken to physical

\footnotetext{
${ }^{\mathrm{a}}$ Corresponding author: sireerat.c@chula.ac.th
}

crosslink with PVA. PLA films reinforced with MFC/PVA were prepared using a solution casting method. The structure and mechanical properties of films were characterized by X-ray diffractometer (XRD) scanning electron microscope (SEM) and tensile testing machine.

\section{Experimental}

\subsection{Materials}

Poly(lactic acid) (PLA) (NatureWork ${ }^{\mathrm{TM}}$ PLA 4043D) in pellet form was purchased from NatureWork ${ }^{\circledR}$ LLC, Minnetonka, MN USA. Corn cobs were obtained from Nakornsawan Province in Thailand. Poly(vinyl alcohol) (PVA) was purchased from Ajax Finechem, Australia.

\subsection{Preparation of MFC}

Ground corn cobs and $1 \mathrm{M} \mathrm{NaOH}$ were heated at $70{ }^{\circ} \mathrm{C}$ with slow stirring for $2 \mathrm{~h}$. The $\mathrm{NaOH}$ extraction was repeated two times and followed by bleaching with $2 \%$ $\mathrm{H}_{2} \mathrm{O}_{2}$ at $80^{\circ} \mathrm{C}$ for $4 \mathrm{~h}$. After cooling, the suspension was filtered and washed with water until $\mathrm{pH} 7$. The obtained cellulose powder was placed in the oven at $60^{\circ} \mathrm{C}$ for $24 \mathrm{~h}$ and kept in desiccator. A cellulose solution was then 
prepared according to Chang et al method [6]. Three grams of cellulose powder was dispersed into $\mathrm{NaOH} /$ urea aqueous solution and was homogenized with high speed homogenizer $(10000 \mathrm{rpm})$ for 20 minutes and then was frozen under refrigeration $\left(-20{ }^{\circ} \mathrm{C}\right)$ for $24 \mathrm{~h}$. The frozen solid was thawed and stirred at room temperature to obtain a transparent MFC solution.

\subsection{MFC/PVA crosslinking}

The MFC/PVA crosslinking was followed by Chang et al method [5]. PVA (3wt\%) was dissolved in $\mathrm{NaOH} /$ urea aqueous solution at $90^{\circ} \mathrm{C}$ for $2 \mathrm{~h}$. The physical crosslinked MFC/PVA was prepared by mixing MFC/PVA solutions in the ratios 70/30 (w/w) and subsequently freezing the mixtures under refrigeration for $24 \mathrm{~h}$, then thawing to room temperature over $2 \mathrm{~h}$. This freezing/thawing cycle was repeated 5 times as shown in Table1.The crosslinked MFC/PVA was kept in refrigerator. The non-crosslinked $\mathrm{MFC} / \mathrm{PVA}$ preparation was similar to the crosslinked MFC/PVA bystirring at room temperature over 2 hbut without freezing/thawing.

\subsection{Preparation of PLA and PLA films reinforced with MFC/PVA}

A $5 \mathrm{wt} \%$ solution ofPLA was prepared by stirring PLA pellets in chloroform at $60{ }^{\circ} \mathrm{C}$ for $2 \mathrm{~h}$ until fully dissolved.The PLA solution was immediately subjected to cast film on glass plates and left at room temperature for $24 \mathrm{~h}$. The PLA filmwas then dried at $60{ }^{\circ} \mathrm{C} 24 \mathrm{~h}$ to evaporate solvent and noted as neat PLA.To prepare the PLA/MFC/PVAfilms, $5 \mathrm{wt} \%$ solution of PLA was mixed with non-crosslinked MFC/PVA $(20 \mathrm{~N})$ and with crosslinked MFC/PVA(20P) in various ratiosas shown in Table 1.The mixtures were homogenized at $4000 \mathrm{rpm}$ for $5 \mathrm{~min}$. The suspension was then sonicated for $10 \mathrm{~min}$ and was immediately casted on a clean glass plate. After removal of the solvent, PLA/MFC/PVAfilms were obtained.

Table 1. Formulations of PLAfilms reinforced with noncrosslinked and physically crosslinkedMFC/PVA (homogenize for $20 \mathrm{~min}$ )through solutioncastingprocess.

\begin{tabular}{|c|c|c|c|}
\hline Formula & $\begin{array}{c}\text { PLA } \\
{[\mathrm{wt} \%]}\end{array}$ & $\begin{array}{c}\text { MFC/PVA } \\
{[\mathrm{wt} \%]}\end{array}$ & $\begin{array}{c}\text { Times of } \\
\text { freezing/ } \\
\text { thawing } \\
\text { cycle }\end{array}$ \\
\hline 99:1 PLA/MFC-20N & 99 & 1 & \\
97:3PLA/MFC-20N & 97 & 3 & - \\
95:5PLA/MFC-20N & 95 & 5 & \\
\hline 99:1PLA/MFC-20P & 99 & 1 & \\
97:3PLA/MFC-20P & 97 & 3 & 5 \\
95:5PLA/MFC-20P & 95 & 5 & \\
\hline
\end{tabular}

\section{Characterization}

X-ray diffraction (XRD) was carried out using the X-ray Power Diffractometer (BRUKER axs, D8). Powder samples were scanned from 3 to 40 degrees 2 theta.

\subsection{Mechanical testing}

Tensile test was done using material testing machine (LLOYD, LF plus). Sample was cut into rectangular pieces about $0.3 \mathrm{~mm}$ thick, $0.5 \mathrm{~mm}$ wide and $5 \mathrm{~mm}$ long. The sample gauge length was $25 \mathrm{~mm}$ and the testing speed at $1 \mathrm{~mm} / \mathrm{min}$. The results were taken as an average of five tests.

\subsection{Morphology}

Morphology of film samples was examined using a scanning electron microscopy (SEM, Jeol JSM 6400) at $15 \mathrm{kV}$ with resolution of $1000 \mathrm{X}$. The neat PLA and PLA/MFA/PVA film samples were analyzed.

\section{Results and Discussion}

\subsection{Structure of corn cob cellulose}

XRD patterns of purified corn cob cellulose and ground corn cobs are shown in Figure 1. The both XRD patterns show characteristic peak of cellulose I at 16 and 22 degrees two theta. Thepattern of ground corn cobs is similar tothe purified corn cob cellulose but broader due

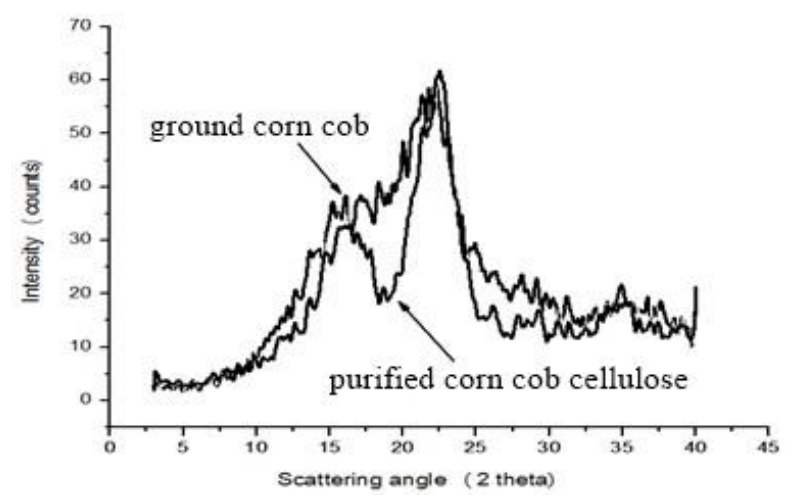

to the presence of non-crystalline hemicelluloses and lignin [7].

Figure 1. X-ray diffraction patterns of ground corn cobs and purified corn cob cellulose.

\subsection{Mechanical properties of PLA andPLA films reinforced with MFC/PVA}

Tensile strength, Young's modulus and elongation at break of neat PLA and PLA films reinforced with MFC/PVAfilms are shown in Figure 2.

Tensile strength, Young's modulus and elongation at break of neat PLA were $20.50 \mathrm{MPa}, 740.40 \mathrm{MPa}$ and $21.23 \%$, respectively. After addition of $1 \mathrm{wt} \%$ crosslinked MFC/PVA, tensile strength and elongation at break increased to $26.21 \mathrm{MPa}$ and $23.21 \%$ respectively but Young's modulus did not improve. This indicated that the 
addition of $1 \mathrm{wt} \%$ crosslinked MFC/PVArendered good interaction between the PLA and MFC which resulted in better tensile strength than that of neat PLA. However, the addition of 3 and $5 \mathrm{wt} \%$ crosslinked MFC/PVA led to a decrease in both tensile strength and elongation at break. This may be attributed to aggregation of the MFC due to the filler-filler interaction becomes greater than filler matrix interaction [1].

The effect of crosslinking MFC using PVA on mechanical properties indicated that crosslinking can improve tensile strength of PLA films because inter- and intra-molecular hydrogen bonds between MFC and PVA molecular chains were strong. These results demonstrated that MFC and PVA molecular chains had been compactly accumulated when freezing/thawing cycles were completed. This led to a decrease in the free volumes and a high strength [5].

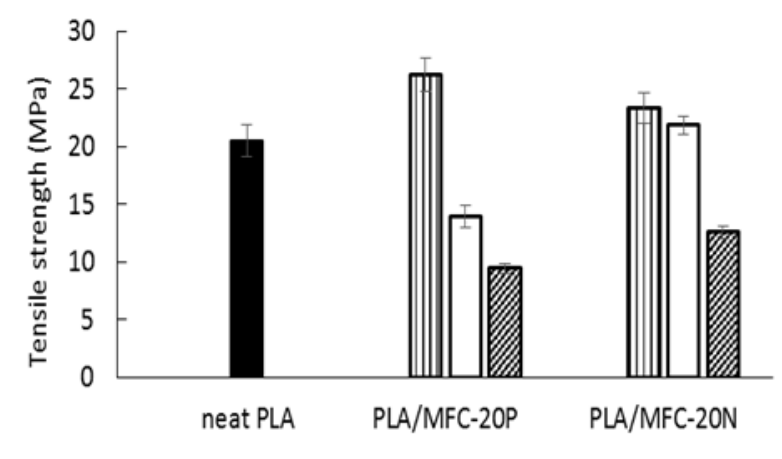

100:0 प99:1 व97:3 ख95:5

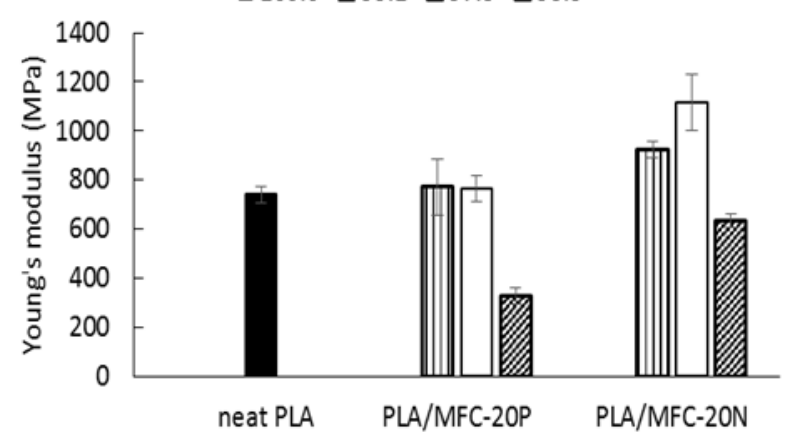

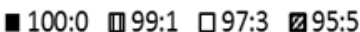

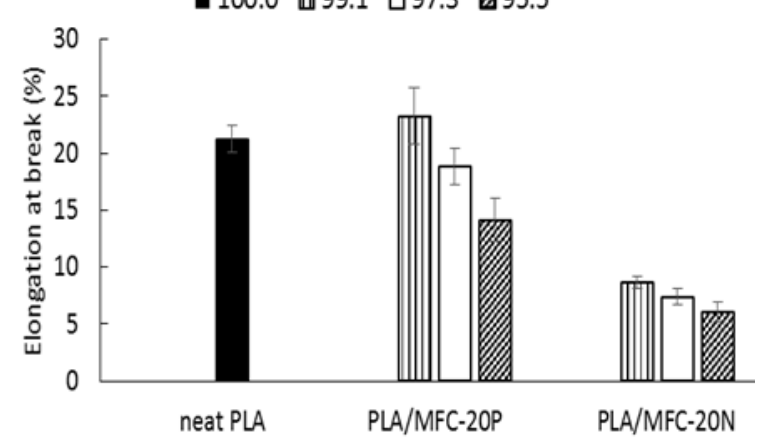

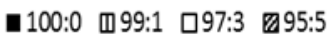

Figure 2. Mechanical properties of neat PLA and PLA reinforced with non-crosslinked and crosslinked MFC/PVA films.

\subsection{Morphology}

The morphology of fractured cross-sectional surfaces of PLA and PLA films reinforced with crosslinked MFC/PVA was studied by SEM as shown in Figure 3. The neat PLA (Figure 3a.) is categorized as ductile fracture because the fibrils remained on the fracture surface [1]. After crosslinkedMFC/PVA added into PLA, the fracture surface showed irregular protrusion and holes as indicated by white arrow in Figure 3b.-3d.This indicated abrittle fracture. The fracture surface of PLA films reinforced with crosslinked MFC/PVA at the ratios of 99:1 (Figure 3b) seems similar to neat PLA.Thisreveals good dispersion and adhesion between crosslinked MFC/PVA and PLA matrix. The SEM results agreed with the mechanicalproperties of composite films $[1,8]$.
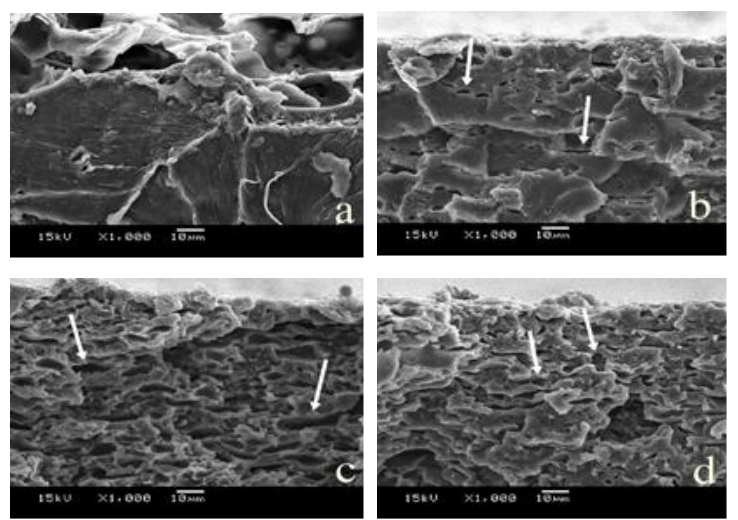

Figure 3. SEM micrographs of the fracture surface of PLA/MFC/PVA films (a) neat PLA (b) 99:1 PLA/MFC-20P (c) 97:3 PLA/MFC-20P and (d) 95:5 PLA/MFC-20P

\section{Summary}

Cellulose was extracted from corn cobs by successive hot $\mathrm{NaOH}$ solution and followed by $\mathrm{H}_{2} \mathrm{O}_{2}$ bleaching. The transparent microfibrillated cellulose (MFC) solution was prepared by dispersing extracted cellulose powder using $\mathrm{NaOH} /$ urea aqueous solution. To improve strength of MFC added to PLA, MFC was then taken to physical crosslink with PVA under freezing/thawing cycles. PLA films reinforced with non-crosslinked MFC/PVA and with crosslinked MFC/PVA were prepared using a solution casting method. It was found that PLA films reinforced with crosslinked MFC/PVA at $1 \mathrm{wt} \%$ has greater tensile strength than that of reinforced with noncrosslinked MFC/PVA at the same contents. However, when MFC contents increase, tensile strength decreased due to the filler-filler interactionwhich was found both in PLA fims reinforced with non-crosslinked and crosslinked MFC/PVA. Morphology of fracture surfaces reveals good dispersion and adhesion between $1 \%$ crosslinked MFC/PVA and PLA matrix. 


\section{Acknowledgements}

This research has been supported by the Ratchaphiseksomphot Endowment Fund 2013 of Chulalongkorn University (CU-56-416-AM).

\section{References}

1. M.K. Mohamad Haafiz, A. Hassan, Z. Zakaria, I.M. Inuwa, M.S. Islam M. Jawaid, Carbohyd. Polym. 98, 139 (2013)

2. M. Jonoobi, J. Harun, A.P. Mathew, K. Oksman, Compos. Sci. Technol. 70, 1742 (2010)
3. A.K. Bledzki, A. Jaszkiewicz D. Scherzer, Compos. Part A-Appl. S. 40, 404 (2009)

4. L. Brinchi, F. Cotana, E. Fortunati J.M. Kenny, Carbohyd. Polym. 94, 154 (2013)

5. C. Chang, A. Lue L. Zhang, Macromol. Chem. Physic. 209, 1266 (2008)

6. C. Chang, L. Zhang, J. Zhou, L. Zhang J.F. Kennedy, Carbohyd. Polym. 82, 122 (2010)

7. R.L. Shogren, S.C. Peterson, K.O. Evans J.A. Kenar, Carbohyd. Polym. 86, 1351 (2011)

8. P. Qu, Y. Gao, G. F Wu L. P. Zhang, BioRes. 5, $1811(2010)$ 